\title{
Abelian simply transitive affine groups of symplectic type
}

\author{
Oliver Baues * \\ Departement Mathematik \\ ETH-Zentrum \\ Rämistrasse 101 \\ CH-8092 Zürich
}

Vicente Cortés ${ }^{\dagger}$

Mathematisches Institut

Universität Bonn

Beringstraße 1

D-53115 Bonn

December 7, 2001

\begin{abstract}
The set of all Abelian simply transitive subgroups of the affine group naturally corresponds to the set of real solutions of a system of algebraic equations. We classify all simply transitive subgroups of the symplectic affine group by constructing a model space for the corresponding variety of solutions. Similarly, we classify the complete global model spaces for flat special Kähler manifolds with a constant cubic form.
\end{abstract}

Keywords: affine transformations, flat symplectic connections, special Kähler manifolds

MSC 2000: 22E25, 22E45, 53C26

\section{Introduction}

Affine groups acting simply transitively on real affine space have been much studied in the literature. (See e.g. the seminal paper $\mathbb{A}]$, or $\mathbb{S}$ for a different point of view.) In

*e-mail: oliver@math.ethz.ch

†e-mail: vicente@math.uni-bonn.de

This work was supported by FIM (ETH Zürich) and MPI für Mathematik (Bonn). 
this paper we study the case of Abelian groups which act by symplectic transformations. Our interest is to describe the properties of the algebraic variety of simply transitive Abelian affine groups of symplectic type. By doing so, we solve the classification problem for these groups up to a linear action on a well understood algebraic space. To put this into perspective we mention that in general Abelian simply transitive groups of affine motions are classified only in low dimensions. (See DO for some recent contribution to this problem.) On the other hand, it is well known that the group of translations is the only simply transitive Abelian group with the property that its linear part preserves a nondegenerate symmetric bilinear form. We mention further that (as follows for example from [FGH, Theorem 6.11,7.1]) the classification of Abelian simply transitive affine groups of symplectic type essentially implies the classification of flat affine tori with a parallel symplectic form up to symplectic affine diffeomorphisms.

Our particular motivation to consider Abelian simply transitive symplectic affine groups stems from special Kähler geometry. Special Kähler geometry is a particular type of geometry which arises in certain supersymmetric field theories, see e.g. [E] and the literature cited therein. A special Kähler manifold is a (pseudo-) Kähler manifold $(M, g)$ which has as additional geometric datum a torsionfree flat connection $\nabla$ which satisfies certain compatibility conditions with respect to $g$. A special Kähler manifold is called flat if the Levi-Civita connection $D$ of the special Kähler metric $g$ is flat. In this paper, we are concerned with the existence and construction of flat special Kähler manifolds.

Clearly, if a Kähler manifold is flat, it is locally modelled on the vector space $\mathbb{C}^{n}$ endowed with a Hermitian inner product, and therefore it is locally trivial as a Kähler manifold. We say that a special Kähler manifold is locally trivial if as a special Kähler manifold it is locally equivalent to a Hermitian vector space. This is the case precisely if $\nabla=D$. It may seem, at first sight, a bit surprising that there do exist flat special Kähler manifolds which are not locally trivial as special Kähler manifolds. In fact, we show that if the special Kähler metric is definite, then flatness implies local triviality. In particular, any simply connected and complete flat special Kähler manifold with a positive definite metric is equivalent to a positive definite Hermitian vector space.

It is well known that special Kähler manifolds arise locally from holomorphic potentials. As it turns out, flat special Kähler manifolds correspond to those holomorphic potentials which satisfy a specific algebraic constraint on their third derivatives. (A priori, flatness is a constraint on the fourth order jet of the potential.) We may interpret this constraint as an associativity condition on an associated bilinear product on the tangent 
spaces of a special Kähler manifold. This shows, by the way, a close structural similarity between flat special Kähler manifolds and Frobenius manifolds. In the non-definite case, the algebraic constraint on cubic tensors admits a non-trivial variety of solutions which we describe as certain bundles over Grassmannians. This enables us to locally classify flat special Kähler manifolds with constant cubic form. In particular, the points of the above solution variety correspond to the complete simply connected global models of such manifolds. These spaces are complete with respect to both the metric connection $D$, and the affine connection $\nabla$. All of them arise from certain Abelian simply transitive affine groups of symplectic type.

\section{Contents}

$\begin{array}{llr}1 & \text { Introduction } & 1\end{array}$

1.1 Statement of main results and outline of the paper f . . . . . . . . . 3

$\begin{array}{lll}2 & \text { Abelian simply transitive affine groups } & 6\end{array}$

2.1 The variety of simply transitive groups $\ldots \ldots \ldots \ldots \ldots$

2.2 The orthogonal and symplectic cases $\ldots \ldots \ldots \ldots$

$\begin{array}{lll}3 & \text { Flat special Kähler manifolds } & 13\end{array}$

3.1 The variety $C_{J}(\mathfrak{s p}(V)) \ldots \ldots \ldots \ldots \ldots \ldots$

3.2 Local characterization of flat special special Kähler manifolds . . . . . . . 18

3.3 Examples with constant cubic form . . . . . . . . . . . . . . . 20

\subsection{Statement of main results and outline of the paper}

The variety of Abelian simply transitive groups The set of simply transitive Abelian groups which act affinely on a vector space $V$ with linear part in a prescribed linear Lie group $G \subset \mathrm{GL}(V)$ constitutes in a natural way a real affine variety. To explain this we show that this set corresponds to the solutions $C(\mathfrak{g})$ of a system of linear and quadratic equations in the first prolongation

$$
\mathfrak{g}^{(1)}:=V^{*} \otimes \mathfrak{g} \cap S^{2}\left(V^{*}\right) \otimes V
$$

of the Lie algebra $\mathfrak{g}=$ Lie $G$. This is done in section 2.1. 
Our guiding principle is that we want not only to write down the equations defining this variety, namely,

$$
C(\mathfrak{g})=\left\{S \in \mathfrak{g}^{(1)} \mid \operatorname{tr} S_{X}=0 \quad \text { and } \quad\left[S_{X}, S_{Y}\right]=0 \quad \text { for all } \quad X, Y \in V\right\}
$$

but want also to solve them by some explicit construction. This is carried out in section 2.2.

We need to introduce some notation to explain our result for $\mathfrak{g}=\mathfrak{s p}(V)$. Let $V$ be a vector space with a nondegenerate alternating product $\omega, \operatorname{dim} V=2 n$. A subspace $U$ of $V$ is called Lagrangian if $U$ is maximally isotropic with respect to $\omega$. For $S \in \mathfrak{g}^{(1)}$ we define the support of $S$ by

$$
\Sigma_{S}:=\operatorname{span}\left\{S_{X} Y \mid X, Y \in V\right\}
$$

The variety $C(\mathfrak{s p}(V))$ is naturally stratified by the dimension of the support. The symplectic form $\omega$ allows the identification of $S^{3} V$ with a subspace of $V^{*} \otimes V^{*} \otimes V$ and a corresponding notion of support for elements of $S^{3} V$. For a subspace $W \subset V$ we define

$$
S^{3} W_{\text {reg }}:=\left\{S \in S^{3} W \mid \Sigma_{S}=W\right\}
$$

The first result is (compare Theorem 9):

Theorem 1 Let $U \subset V$ be a Lagrangian subspace. There is a one-to-one correspondence between Abelian simply transitive affine groups of symplectic type up to conjugation in the affine symplectic group and elements of $S^{3} U$ up to $\operatorname{Sp}(V)$-equivalence (two elements of $S^{3} U$ are $\operatorname{Sp}(V)$-equivalent if they lie on the same $\operatorname{Sp}(V)$-orbit in $\left.S^{3} V\right)$.

Let $\mathcal{U}_{k} \rightarrow G_{k}^{0}(V)$ denote the universal (tautological) vector bundle over the Grassmannian $G_{k}^{0}(V)$ of $k$-dimensional isotropic subspaces, and $S^{3} \mathcal{U}_{k}$ the third symmetric power of this bundle. We prove then (compare Theorem 10):

Theorem 2 The $k$-th stratum $C(\mathfrak{s p}(V))_{k}$ of the variety $C(\mathfrak{s p}(V))$ naturally identifies with the Zariski open subbundle

$$
\bigcup_{W \in G_{k}^{0}(V)} S^{3} W_{r e g}
$$

of the vector bundle $S^{3} \mathcal{U}_{k}$.

Now the classification for Abelian simply transitive affine groups of symplectic type reads as follows (compare Theorem 10, Corollary 4): 
Corollary 1 There is a natural one-to-one correspondence between Abelian simply transitive affine groups of symplectic type with (maximal) translation subgroup of dimension $2 n-k$ up to conjugation, and homogeneous cubic polynomials in $S^{3} W_{\text {reg }}$ up to the linear action of $\mathrm{GL}(\mathrm{W})$, where $W$ is a $k$-dimensional isotropic subspace of $V$.

In particular, every such group contains an $n$-dimensional subgroup of translations.

Special Kähler manifolds Let us turn now to the second part of our article which starts in section 3. A special Kähler manifold is a (possibly indefinite) Kähler manifold $(M, J, g)$ endowed with a flat torsionfree connection $\nabla$ such that $\nabla J$ is symmetric and $\nabla \varnothing=0$, where $\varnothing=g(\cdot, J \cdot)$ is the symplectic (Kähler) form $[\mathbb{F}]$. A special Kähler manifold is called flat if the Levi-Civita connection $D$ is flat. The simplest example of such a manifold is obtained by taking a (pseudo-) Hermitian vector space $\mathbb{C}^{n}$, so that the flat Levi-Civita connection $D$ and the symplectic connection $\nabla$ coincide. Henceforth a special Kähler manifold is called trivial if $D=\nabla$. We show:

Theorem 3 Any flat special Kähler manifold with a definite metric is trivial.

This result is obtained by considering the algebraic constraints on the difference tensor $S=D-\nabla$ of a flat special Kähler manifold. Let $\left(V=\mathbb{C}^{n}, J, g\right)$ be the standard (pseudo-) Hermitian vector space of complex signature $(p, q), p+q=n$. We show that the tensor field $S$ takes values in a subvariety $C_{J}(\mathfrak{s p}(V))$ of the cone $C(\mathfrak{s p}(V))$. In section 3.1 we obtain a description of the variety $C_{J}(\mathfrak{s p}(V))$ in a way analoguous to Theorem 1 and Theorem 2 above. These results allow for the local classification of special Kähler manifolds in section 3.2 as follows:

Theorem 4 Let $f$ be a holomorphic function defined on an open subset $M \subset V$. Assume that the pointwise support of the cubic tensor field $\partial^{3} f: M \rightarrow S^{3,0} V^{*}$ defined by the holomorphic third partial derivatives of $f$ is isotropic and put $S_{f}:=\partial^{3} f+\overline{\partial^{3} f} \in S^{3} V^{*}$. Then $M_{f}:=\left(M, J, g, \nabla:=D+S_{f}\right)$ is a flat special Kähler manifold and any flat special Kähler manifold arises locally in this way.

As a special case of the theorem we can describe the class of flat special Kähler manifolds with constant cubic form, i.e., those manifolds which satisfy $D S=0$. They admit a model with an Abelian simply transitive group of automorphisms. 
Theorem 5 Let $W \subset V$ be a complex isotropic subspace, and $f: V \rightarrow \mathbb{C}$ a holomorphic cubic polynomial which identifies with an element of $S^{0,3} \mathrm{~W}$ under the canonical identification $S^{0,3} V^{*} \cong S^{3,0} \mathrm{~V}$. Then the manifold $V_{f}$, defined in the previous theorem, is a flat special Kähler manifold with constant cubic form and complete connections $D$ and $\nabla$. Moreover, any flat special Kähler manifold with constant cubic form is locally equivalent to an open subset of some space $V_{f}$.

The result may be refined a bit. We assume that $(V, J, g)$ admits a complex Lagrangian subspace $U$. Then any flat special Kähler manifold with constant cubic form and without trivial factor may be obtained by some $S \in S^{0,3} U_{\text {reg }}$, and we obtain a bijection between the orbits of the group $\mathrm{GL}_{\mathbb{C}}(U)$ on $S^{0,3} U_{\text {reg }}$ and equivalence classes of germs of flat special Kähler manifolds with constant cubic form and without trivial factor. (See Theorem 17.) Any such manifold has complex signature $(m, m), m=\operatorname{dim}_{\mathbb{C}} U$.

\section{Abelian simply transitive affine groups}

Let $V$ be a real vector space and $G \subset \mathrm{GL}(V)$ any Lie subgroup. We denote by $\operatorname{Aff}_{G}(V) \subset$ $\operatorname{Aff}(V)$ the group of affine transformations with linear part in $G$. Connected Lie subgroups of $\operatorname{Aff}_{G}(V)$ are called affine groups of type $G$. Lie subalgebras of aff $\mathfrak{g}(V)=$ Lie $\operatorname{Aff}_{G}(V)$ are called affine Lie algebras of type $\mathfrak{g}=$ Lie $G$. We are interested in Abelian affine groups $H$ of type $G$ acting simply transitively on $V$. This means that the orbit map $\varphi: H \ni h \mapsto h 0 \in V$ is a diffeomorphism. More generally, we will consider almost simply transitive groups, i.e. groups for which $\varphi$ is an immersion. The corresponding Lie subalgebras $\mathfrak{h}=$ Lie $H \subset \operatorname{aff}_{\mathfrak{g}}(V)$ are almost simply transitive. This means that the linear map $\phi: \mathfrak{h} \ni X \mapsto X 0 \in V$ is an isomorphism. In fact, this map is the differential of the orbit map $\varphi$, which is an immersion since $H$ is almost simply transitive. The Lie algebras of simply transitive groups satisfy a stronger condition. They are simply transitive, which means that for all $v \in V$ the linear map $\mathfrak{h} \ni X \mapsto X v \in T_{v} V \cong V$ is an isomorphism. The next proposition reduces the study of Abelian (almost) simply transitive affine groups to that of Abelian (almost) simply transitive affine Lie algebras.

Proposition 1 The Lie-functor from the category of Lie groups to the category of Lie algebras induces a bijection between the set of Abelian simply transitive affine groups of type $G$ and the set of Abelian simply transitive affine Lie algebras of type $\mathfrak{g}=$ Lie $G$. The same is true for almost simply transitive affine groups and Lie algebras. 
Proof. We have seen that the Lie algebra of an Abelian (almost) simply transitive group of type $G$ is an Abelian (almost) simply transitive Lie algebra of type $\mathfrak{g}$. It is clear that the Abelian affine group $H$ of type $G$ generated by an Abelian almost simply transitive Lie algebra $\mathfrak{h}$ of type $\mathfrak{g}$ is almost simply transitive. It remains to show that the group generated by a simply transitive Lie algebra $\mathfrak{h}$ is simply transitive. From the fact that $\mathfrak{h}$ is simply transitive it follows that all orbits of $H$ are open. $V$ being connected, this implies that $H$ is transitive. So we have a diffeomorphism $V \stackrel{\sim}{\rightarrow} H / H_{v}$, where $H_{v}$ is the stabilizer of a point $v \in V$. Moreover $H_{v}$ is discrete and $H \rightarrow H / H_{v} \cong V$ is a covering of the simply connected manifold $V$. This implies that $H_{v}$ is trivial and hence that $H$ is simply transitive.

Let us denote by $A^{\prime}(G)$ (respectively $A^{\prime}(\mathfrak{g})$ ) the set of Abelian almost simply transitive affine groups of type $G$ (respectively the set of Abelian almost simply transitive affine Lie algebras of type $\mathfrak{g})$. The subsets consisting of simply transitive groups and Lie algebras are denoted by $A(G)$ and $A(\mathfrak{g})$, respectively. Note that by the previous proposition we can identify $A(G)=A(\mathfrak{g})$ and $A^{\prime}(G)=A^{\prime}(\mathfrak{g})$.

\subsection{The variety of simply transitive groups}

Let $\iota: \mathfrak{h} \hookrightarrow \operatorname{aff} \mathfrak{g}(V)$ be the canonical inclusion map associated to a Lie algebra $\mathfrak{h} \in A^{\prime}(\mathfrak{g})$. It gives rise to a injective linear map $\rho=\rho_{\mathfrak{h}}: V \longrightarrow \operatorname{aff}_{\mathfrak{g}}(V)$ by

$$
\rho:=\iota \circ \phi^{-1}
$$

where $\phi$ is the differential of the orbit map $\varphi: H \ni h \mapsto h 0 \in V$ at the identity. We remark that, since $\mathfrak{h}$ is assumed to be an Abelian Lie algebra, the linear map $\rho$ : $V \longrightarrow \operatorname{aff}_{\mathfrak{g}}(V)$ is in fact a homomorphism of Lie Algebras. The affine Lie algebra aff $\mathfrak{g}(V)=\mathfrak{g}+V$ is the semidirect sum of the linear Lie algebra $\mathfrak{g}$ and the ideal $V$ of infinitesimal translations. We denote an element of this semidirect sum as a pair $(S, t)$, where $S \in \mathfrak{g}$ is the linear part and $t \in V$ the translational part. In particular we can write the monomorphism $\rho=\rho_{\mathfrak{h}}$ in the form

$$
\rho(X)=\left(S_{X}, t_{X}\right), \quad X \in V,
$$

where $S: V \rightarrow \mathfrak{g}$ and $t: V \rightarrow V$ are linear maps.

Proposition 2 Let $\rho=(S, t): V \longrightarrow \operatorname{aff} \mathfrak{g}(V)=\mathfrak{g}+V$ be the monomorphism associated to an Abelian almost simply transitive affine Lie algebra of type $\mathfrak{g}$. Then $t=i d_{V}$ and $S \in V^{*} \otimes \mathfrak{g}$ satisfies: 
(i) $S_{X} Y=S_{Y} X$, for all $X, Y \in V$.

(ii) $\left[S_{X}, S_{Y}\right]=0$, for all $X, Y \in V$.

Conversely, any $S \in V^{*} \otimes \mathfrak{g}$ satisfying (i) and (ii) defines a monomorphism $\rho=\left(S, i d_{V}\right)$ : $V \longrightarrow \operatorname{aff}_{\mathfrak{g}}(V)$ onto an Abelian almost simply transitive affine Lie algebra $\mathfrak{h}=\rho(V)$ of type $\mathfrak{g} . \mathfrak{h}$ is simply transitive if and only if

(iii) $\operatorname{tr} S_{X}=0$, for all $X \in V$.

Proof. Let us first check that $t=i d_{V}$. This follows from

$$
t_{X}=\rho(X) 0=\iota\left(\phi^{-1}(X)\right) 0=\phi^{-1}(X) 0=\phi\left(\phi^{-1}(X)\right)=X .
$$

Equations (i-ii) express that $\rho: V \rightarrow \operatorname{aff} \mathfrak{g}(V)$ is a homomorphism of Lie algebras.

Now we show that (iii) is equivalent to the image of $\rho$ being a simply transitive Lie algebra. Let us first assume that $\mathfrak{h}=\rho(V)$ is a simply transitive Lie algebra. Let $H$ be the corresponding simply transitive subgroup of Aff(V). For $h \in H$ we consider the Jordan-decomposition $h=h_{s} h_{u}$ inside the real linear algebraic group Aff(V). Since $H$ is Abelian, $T=\left\{h_{s} \mid h \in H\right\}$ is a group of semisimple operators which centralizes $H$. By semi-simplicity $T$ has a fixed point on $V$. But since $T$ centralizes the transitive group $H$ it must be trivial. Therefore $H$ is unipotent, and in particular the linear parts of the elements of $H$ are unipotent. This implies (iii).

Conversely, we prove that (i)-(iii) implies that $\mathfrak{h}$ is simply transitive. We have to show that the linear map $V \ni X \mapsto S_{X} v+X \in V$ is an isomorphism for all $v \in V$. Therefore it is sufficient to prove that the map $X \mapsto S_{X} v=S_{v} X$ is nilpotent, for all $v \in V$. Let us show that $\operatorname{tr} S_{v}^{k}=0$ for all $k=1,2, \ldots$ Because of (i) and (ii) we have $S_{v}^{k}=S_{w}$, where $w:=S_{v}^{k-1} v$. Now $\operatorname{tr} S_{v}^{k}=0$ follows from (iii). This shows that $S_{v}$ is nilpotent, for all $v \in V$.

We recall [K] that the first prolongation of a Lie algebra $\mathfrak{g} \subset V^{*} \otimes V$ is defined as

$$
\mathfrak{g}^{(1)}:=V^{*} \otimes \mathfrak{g} \cap S^{2}\left(V^{*}\right) \otimes V
$$

Note that the equation (i) says that $S$ is an element of $\mathfrak{g}^{(1)}$. We denote by $C^{\prime}(\mathfrak{g}) \subset \mathfrak{g}^{(1)}$ the cone defined by the system (ii) of homogeneous quadratic equations. It is an affine real algebraic variety. The condition (iii) defines a Zariski closed subset $C(\mathfrak{g}) \subset C^{\prime}(\mathfrak{g})$. In fact, $C(\mathfrak{g})=C^{\prime}\left(\mathfrak{g}_{0}\right)=C^{\prime}(\mathfrak{g}) \cap \mathfrak{g}_{0}^{(1)}$, where $\mathfrak{g}_{0}=\{A \in \mathfrak{g} \mid \operatorname{tr} A=0\}$. 
Theorem 6 The correspondence $H \mapsto S$, where $S \in \mathfrak{g}^{(1)}$ is the linear part of $\rho_{\mathfrak{h}}$, induces bijections $A^{\prime}(G) \stackrel{\sim}{\rightarrow} C^{\prime}(\mathfrak{g})$ and $A(G) \stackrel{\sim}{\rightarrow} C(\mathfrak{g})$. This defines on the set $A^{\prime}(G)$ of Abelian almost simply transitive affine Lie groups of type $G$ the structure of an affine cone over a quadratic projective real algebraic variety. The subset $A(G) \subset A^{\prime}(G)$ consisting of simply transitive groups is a closed subvariety defined by a system of linear equations. Under the above identification, the action of $g \in G$ on $A^{\prime}(G)$ by conjugation corresponds to the linear action $\mathfrak{g}^{(1)} \ni S \mapsto g \cdot S \in \mathfrak{g}^{(1)}$, where $(g \cdot S)_{X}:=g S_{g^{-1} X} g^{-1}$.

Proof. This follows essentially from Proposition 2. We check the formula for the $G$-action on $C^{\prime}(\mathfrak{g}) \subset \mathfrak{g}^{(1)}$. From the definition of $g \cdot S$ it follows that the homomorphism $\left((g \cdot S), i d_{V}\right)$ has the same image as

$$
X \mapsto\left(g S_{X} g^{-1}, g X\right)=g \rho_{\mathfrak{h}}(X) g^{-1}
$$

namely the conjugated Lie subalgebra $\operatorname{Ad}_{g}(\mathfrak{h})=g \mathfrak{h} g^{-1} \subset \operatorname{aff} \mathfrak{g}(V)$. This shows that $\left((g \cdot S), i d_{V}\right)=\rho_{\operatorname{Ad}_{g}(\mathfrak{h})}$ and hence that $S^{g H g^{-1}}=g \cdot S^{H}$.

Now we specialize to the unimodular case, i.e. we assume that $G \subset \mathrm{SL}(V)$.

Corollary 2 An Abelian almost simply transitive affine group $H$ of of type $\mathrm{SL}(V)$ is simply transitive. In particular, $A(G)=A^{\prime}(G)$ and $C(\mathfrak{g})=C^{\prime}(\mathfrak{g})$ for all $G \subset \mathrm{SL}(V)$.

Proof. This is a direct consequence of Proposition 2.

In the following section we specialize our discussion to the case where $G$ preserves a (possibly indefinite) scalar product or a symplectic form on $V$.

\subsection{The orthogonal and symplectic cases}

Theorem 7 Let $V$ be a pseudo-Euclidean vector space and $G \subset \mathrm{SO}(V)$ a Lie subgroup. Then $A(G)$ is a point. In other words, the translation group $V$ is the only Abelian simply transitive affine group of type $G$.

Proof. By Theorem 6 we know that $A(G) \subset \mathfrak{g}^{(1)} \subset \mathfrak{s o}(V)^{(1)}$. Now the theorem follows from the fact that $\mathfrak{s o}(V)^{(1)}=0$.

Let now $V$ be a symplectic vector space. The symplectic case is more interesting since the first prolongation of the symplectic Lie algebra is nontrivial. In fact, using the symplectic form $\varnothing$ on $V$ we identify $V$ with $V^{*}$ via $v \mapsto \emptyset v:=\varnothing(v, \cdot)$. This induces 
identifications of the Lie algebra $\mathfrak{s p}(V)$ with the symmetric square $S^{2} V^{*}$ and of its first prolongation with the symmetric cube $S^{3} V^{*}$, since

$$
\mathfrak{s p}(V)^{(1)}=V^{*} \otimes \mathfrak{s p}(V) \cap S^{2} V^{*} \otimes V=V^{*} \otimes S^{2} V^{*} \cap S^{2} V^{*} \otimes V^{*}=S^{3} V^{*} .
$$

Explicitly, the identification of $S \in \mathfrak{s p}(V)^{(1)}$ with a totally symmetric trilinear form is given by $(X, Y, Z) \mapsto \varnothing\left(S_{X} Y, Z\right)$. Notice that $S^{3} V^{*}$ is also the vector space of homogeneous cubic polynomials on $V$. The preceding discussion together with Corollary 2 establishes therefore the following theorem.

Theorem 8 The correspondence $H \mapsto S$ of Theorem 0 identifies $A(\operatorname{Sp}(V))$ with a quadratic cone $C(\mathfrak{s p}(V))$ in the vector space of cubic forms $S^{3} V^{*}$.

In the following we identify of $S^{3} V^{*}$ with $S^{3} V$ by means of $\varnothing$. Next we want to give an explicit construction of the solutions of the quadratic equations defining the affine variety $C(\mathfrak{s p}(V)) \subset S^{3} V$. We recall that the group $G$ acts by conjugation on $A(\mathfrak{g})$. By Theorem 6, under the identification $A(\mathfrak{g})=C(\mathfrak{g}) \hookrightarrow \mathfrak{g}^{(1)}$, this action is induced by the natural linear action on $\mathfrak{g}^{(1)}$. In the case of $G=\operatorname{Sp}(V)$ this is the standard representation on $S^{3} V^{*} \cong S^{3} V$. We are also interested in the orbit space $\bar{A}(\mathfrak{g}):=A(\mathfrak{g}) / G$, which is the space of conjugacy classes of Abelian simply transitive affine Lie algebras of type $G$. We have a natural inclusion $\bar{A}(\mathfrak{s p}(V)) \subset S^{3} V / \operatorname{Sp}(V)$.

Theorem 9 Let $U \subset V$ be a Lagrangian subspace of a symplectic vector space $V$. Any $\operatorname{Sp}(V)$-orbit in $C(\mathfrak{s p}(V)) \subset S^{3} V$ intersects the subspace $S^{3} U \subset S^{3} V$. Moreover

$$
C(\mathfrak{s p}(V))=\bigcup_{U} S^{3} U \subset S^{3} V,
$$

where the union is over all Lagrangian subspaces $U \subset V$.

Proof. Let $S \in S^{3} U$. Then the map $X \mapsto S_{X}$ takes values in $S^{2} U \subset S^{2} V=\mathfrak{s p}(V)$. To see that $S \in C(\mathfrak{s p}(V))$ it is sufficient to observe that, since $U$ is isotropic, $S^{2} U \subset S^{2} V=$ $\mathfrak{s p}(V)$ is an Abelian subalgebra of linear operators.

Now let $S \in C(\mathfrak{s p}(V))$. We have to show that there exists a Lagrangian subspace $U \subset V$ such that $S \in S^{3} U$. We prove first that the support

$$
\Sigma_{S}:=\operatorname{span}\left\{S_{X} Y \mid X, Y \in V\right\}
$$


of $S$ is isotropic. This implies $\Sigma_{S} \subset U$ for some Lagrangian subspace $U \in V$. It is sufficient to check that $S_{X}^{2}=0$ for all $X \in V$. In fact, by polarization this implies $S_{X} S_{Y}=0$ for all $X, Y \in V$ and hence

$$
\varnothing\left(S_{X} Y, S_{Z} W\right)=-\varnothing\left(Y, S_{X} S_{Z} W\right)=0
$$

The claim that $S_{X}^{2}=0$ follows from the next computation.

$$
\begin{gathered}
\emptyset\left(S_{X}^{2} Y, Z\right)=\varnothing\left(S_{Y} S_{X} X, Z\right)=-\varnothing\left(S_{X} X, S_{Y} Z\right)=-\varnothing\left(S_{X} X, S_{Z} Y\right)=\varnothing\left(S_{Z} S_{X} X, Y\right) \\
=\varnothing\left(S_{X}^{2} Z, Y\right)=\varnothing\left(Z, S_{X}^{2} Y\right)=-\varnothing\left(S_{X}^{2} Y, Z\right) .
\end{gathered}
$$

Now the theorem follows from the next lemma.

Lemma 1 Let $S \in S^{3} V$ and $\Sigma_{S} \subset V_{0}$, where $V_{0} \subset V$ is a subspace. Then $S \in S^{3} V_{0}$.

Proof. Let $V_{1} \subset V$ be a complement of $V_{0}$. We denote by $V_{0}^{\wedge}$ and $V_{1}^{\wedge}$ the annihilators of $V_{0}$ and $V_{1}$ in $V^{*}$, respectively, and consider them again as subspaces of $V$. The decomposition

$$
S^{3} V=\oplus_{p+q=3} S^{p} V_{0} \cdot S^{q} V_{1}
$$

gives rise to a decomposition $S=\sum_{p+q=3} S^{p, q}$. For $X, Y \in V_{0}^{\wedge}$ we have $V_{0} \ni S_{X} Y=$ $S_{X}^{0,3} Y+S_{X}^{1,2} Y$ and $S_{X}^{0,3} Y \in V_{1}$. Hence, $S^{0,3}=0$. Similarly, by considering $S_{V_{1}^{\wedge}} V_{0}^{\wedge}$ and $S_{V_{1}^{\wedge}} V_{1}^{\wedge}$ we can conclude that $S^{1,2}=0$ and $S^{2,1}=0$. This shows that $S=S^{3,0} \in S^{3} V_{0}$.

For Lie subgroups of the symplectic group we obtain:

Corollary 3 Let $G \subset \mathrm{Sp}(V)$ be a Lie subgroup. Then

$$
C(\mathfrak{g})=\mathfrak{g}^{(1)} \cap C(\mathfrak{s p}(V))=\bigcup_{U}\left(\mathfrak{g}^{(1)} \cap S^{3} U\right) \subset S^{3} V
$$

where the union is over all Lagrangian subspaces $U \subset V$.

Now we study more closely the cone $C(\mathfrak{s p}(V))$. Let us define

$$
C(\mathfrak{s p}(V))_{k}:=\left\{S \in C(\mathfrak{s p}(V)) \mid \operatorname{dim} \Sigma_{S}=k\right\}
$$

For any isotropic subspace $W \subset V$ we define

$$
S^{3} W_{\text {reg }}:=\left\{S \in S^{3} W \mid \Sigma_{S}=W\right\}
$$

We denote the Grassmannian of isotropic subspaces $W \subset V$ of dimension $k$ by $G_{k}^{0}(V)$. Let $\mathcal{U}_{k} \rightarrow G_{k}^{0}(V)$ be the universal (tautological) vector bundle. 
Theorem 10 The cone $C(\mathfrak{s p}(V))$ has a stratification:

$$
C(\mathfrak{s p}(V))=\bigcup_{k=0}^{n} C(\mathfrak{s p}(V))_{k},
$$

where $2 n=\operatorname{dim} V$. The $k$-th stratum

$$
C(\mathfrak{s p}(V))_{k}=\bigcup_{W \in G_{k}^{0}(V)} S^{3} W_{\text {reg }}
$$

is a Zariski open subbundle of the vector bundle $S^{3} \mathcal{U}_{k}$. The action of $\operatorname{Sp}(V)$ on $C(\mathfrak{s p}(V))$ preserves the stratification and we have the following identification of orbit spaces:

$$
C(\mathfrak{s p}(V))_{k} / \operatorname{Sp}(V)=S^{3} W_{\text {reg }} / \mathrm{GL}(W)
$$

where $W \subset V$ is a fixed $k$-dimensional isotropic subspace.

Proof. It is clear that

$$
C(\mathfrak{s p}(V))=\bigcup_{k=0}^{2 n} C(\mathfrak{s p}(V))_{k} .
$$

It follows from Theorem 9 that the support $\Sigma_{S}$ of any $S \in C(\mathfrak{s p}(V))$ is isotropic. This implies (2.5) and, in particular, that $C(\mathfrak{s p}(V))_{k}=\emptyset$ for all $k>n$. For the identification of orbit spaces it is sufficient to remark that two cubic forms $S$ and $S^{\prime}$ with isotropic support $W$ are related by an element of $\operatorname{Sp}(V)$ if and only if they are equivalent under $\mathrm{GL}(W)$. In fact, since $W$ is isotropic, any element of $\mathrm{GL}(W)$ can be extended to an element of $\operatorname{Sp}(V)$, which preserves $W$. Conversely, any element of $\operatorname{Sp}(V)$ which maps $S$ to $S^{\prime}$ has to preserve $W=\Sigma_{S}=\Sigma_{S^{\prime}}$ and, hence, induces an element of $\operatorname{GL}(W)$, which maps $S$ to $S^{\prime}$.

Corollary 4 Let $H \subset \mathrm{Aff}\left(\mathbb{R}^{2 n}\right)$ be an Abelian simply transitive affine group of symplectic type, $S \in S^{3} V$ the cubic form defined by $H$. Then the subgroup of translations in $H$ is of dimension $2 n-k$, where $k=\operatorname{dim} \Sigma_{S} \leq n$.

Proof. The subgroup of translations in $H$ is the kernel of the map $X \mapsto S_{X}$ which coincides with the kernel of $S$ as an element of $S^{3} V^{*}$. Since this is precisely the orthogonal complement of $\Sigma_{S}$ with respect to $\omega$, the dimension is $2 n-k$. Since $\Sigma_{S}$ is isotropic, $k \leq n$. 


\section{$3 \quad$ Flat special Kähler manifolds}

Definition 1 A (possibly indefinite) Kähler manifold is a (possibly indefinite) Riemannian manifold $(M, g)$ endowed with a parallel complex structure $J$, i.e. DJ $=0$ for the Levi-Civita connection $D$ of $g$, so that $g$ is Hermitian with respect to $J$.

To any Kähler manifold $(M, g, J)$ we can canonically associate the parallel symplectic form $\varnothing=g(J \cdot, \cdot)$. It is called the Kähler form.

Definition $2 A$ special Kähler manifold is a Kähler manifold $(M, g, J)$ endowed with a torsionfree and flat connection $\nabla$ such that

(i) $\nabla \varnothing=0$ and

(ii) $\nabla J$ is symmetric, i.e. $\left(\nabla_{X} J\right) Y=\left(\nabla_{Y} J\right) X$ for all vector fields $X, Y$.

A special Kähler manifold $(M, g, J, \nabla)$ is called flat if the Levi-Civita connection $D$ of $g$ is flat.

Example: Let $(M, g, J)$ be a flat Kähler manifold, i.e. a Kähler manifold for which the Levi-Civita connection $D$ is flat. Then $(M, g, J, D)$ is a flat special Kähler manifold. Special Kähler manifolds of this type will be called trivial special Kähler manifolds.

The following characterization of trivial special Kähler manifolds is easy to verify:

Proposition 3 Let $(M, g, J, \nabla)$ be a special Kähler manifold and D its Levi-Civita connection. Then the following three conditions are equivalent:

(i) $(M, g, J, \nabla)$ is a trivial special Kähler manifold,

(ii) $\nabla=D$ and

(iii) $\nabla J=0$.

Let us recall now some known results on special Kähler manifolds which relate to flatness. It was proven in $[\mathrm{BC}$ that any simply connected special Kähler manifold $(M, g, J, \nabla)$ of complex dimension $n$ can be canonically immersed into $\mathbb{R}^{2 n+1}$ as a parabolic affine hypersphere with Blaschke metric $g$ and affine connection $\nabla$ (the notions of affine hypersphere, Blaschke metric and affine connection are discussed in detail in [NS]). We recall the following classical theorem of Calabi and Pogorelov [Ca]. 
Theorem 11 If the Blaschke metric $g$ of a parabolic affine hypersphere $M$ of dimension $m$ is definite and complete, then $M$ is affinely congruent to the paraboloid $x^{m+1}=$ $\sum_{i=1}^{m}\left(x^{i}\right)^{2}$ in $\mathbb{R}^{m+1}$. In particular, $g$ is flat.

This implies:

Theorem 12 Any special Kähler manifold $(M, g, J, \nabla)$ with a definite and complete metric $g$ is trivial.

Proof. By [BC], the universal covering is immersed as an affine hypersphere, which by Theorem 11 is a paraboloid. This implies that $\nabla=D$ is the Levi-Civita connection and hence that $(M, g, J, \nabla)$ is trivial.

$\mathrm{Lu}$ [4] proved that any special Kähler manifold $(M, g, J, \nabla)$ with a definite and complete metric $g$ is flat. Completeness is a very strong global assumption. Flatness on the other hand is a strong local assumption. If the metric is definite the following rigidity result holds:

Theorem 13 Any special Kähler manifold $(M, g, J, \nabla)$ with a definite and flat metric is trivial.

A proof will be given in the next section. But let us remark that by the immersion theorem mentioned above Theorem 13 may also be read off from the classification of affine hyperspheres with flat and definite Blaschke metric VLS. Without the assumption that the metric is definite Theorem 13 does not hold. We will show that nontrivial flat indefinite special Kähler manifolds exist and give a local characterization of such manifolds in section 3.2. More specifically, we construct there examples of such manifolds which are geodesically complete with respect to both connections $D$ and $\nabla$.

\subsection{The variety $C_{J}(\mathfrak{s p}(V))$}

Let $(M, g, J, \nabla)$ be a flat special Kähler manifold. We are interested in the local properties of $M$, and since $(M, g, J)$ is a flat Kähler-manifold we may as well consider $M$ as ball in a standard Hermitian vectors space $(V, J, g)$. The symplectic connection $\nabla$ may be expressed as $\nabla=D+S$, where $S$ is a one-form on $M$ with values in $\mathfrak{s p}(V)$. We can think of it as a map $S: M \rightarrow V^{*} \otimes S^{2} V^{*}$.

Proposition 4 The tensor field S satisfies the following conditions 
(i) $S \in S^{3} V^{*}=\mathfrak{s p}(V)^{(1)}$,

(ii) $\left[S_{X}, S_{Y}\right]=0$ for all $X, Y \in V$,

(iii) $\left(D_{X} S\right) Y=\left(D_{Y} S\right) X$ for all $X, Y \in V$ and

(iv) $S_{X} J=-J S_{X}$ for all $X \in V$.

Conversely, any tensor field satisfying the conditions (i-iv) defines a flat special Kähler manifold $(M, g, J, \nabla:=D+S)$.

Notice that (i-ii) say that $S$ has values in the cone $C(\mathfrak{s p}(V))$.

Proof. The first condition is equivalent to the vanishing of the torsion of $\nabla$. We claim that the Levi-Civita connection can be expressed as:

$$
D=\nabla-\frac{1}{2} J \nabla J
$$

The right-hand side is a torsionfree connection, by the symmetry of $\nabla J$. Moreover it preserves $J$ and hence the metric $g=\varnothing(\cdot, J \cdot)$ :

$$
\begin{aligned}
\left(\nabla-\frac{1}{2} J \nabla J\right)_{X} J & =\nabla_{X} J-\frac{1}{2}\left[J \nabla_{X} J, J\right] \\
& =\nabla_{X} J-\frac{1}{2} J\left(\nabla_{X} J\right) J+\frac{1}{2} J^{2} \nabla_{X} J=\frac{1}{2}\left(\nabla_{X} J-J\left(\nabla_{X} J\right) J\right)=0 .
\end{aligned}
$$

For the last equation we have used that $J^{2}=-i d$ implies

$$
J \nabla_{X} J=-\left(\nabla_{X} J\right) J .
$$

This proves the formula for the Levi-Civita connection, which we can restate as:

$$
2 S_{X}=J \nabla_{X} J .
$$

From (3.3) and (3.2) we conclude (iv). The remaining equations follow from the flatness of $\nabla$, as we will show now. We compute the curvature $R^{\nabla}$ of $\nabla$ :

$$
\begin{aligned}
R^{\nabla}(X, Y) & =\left[\nabla_{X}, \nabla_{Y}\right]-\nabla_{[X, Y]} \\
& =R^{D}(X, Y)+D_{X}\left(S_{Y}\right)-D_{Y}\left(S_{X}\right)+\left[S_{X}, S_{Y}\right]-S_{[X, Y]} \\
& =\left(D_{X} S\right)_{Y}-\left(D_{Y} S\right)_{X}+\left[S_{X}, S_{Y}\right] .
\end{aligned}
$$

Here we have used that $D$ is flat, i.e. $R^{D}=0$, and torsionfree. Now $\left[S_{X}, S_{Y}\right]$ commutes with $J$, whereas $\left(D_{X} S\right)_{Y}-\left(D_{Y} S\right)_{X}$ anticommutes with $J$, since $S_{X}$ and $S_{Y}$ anticommute with $J$, by (iv), and $D J=0$. This shows that $R^{\nabla}=0$ implies (ii) and (iii). 
Conversely, let $S$ be a tensor field satisfying (i-iv). Then $\nabla=D+S$ is a symplectic torsionfree (i) and by the formula above flat (ii-iii) connection. It only remains to check the symmetry of $\nabla J$. For this we have to compute $\nabla J$ in terms of $S$ arriving again at (3.3):

$$
\nabla_{X} J=D_{X} J+\left[S_{X}, J\right]=\left[S_{X}, J\right] \stackrel{(i v)}{=}-2 J S_{X}
$$

Now the symmetry of $S$ implies that of $\nabla J$.

The previous proposition shows that it is important to understand the following closed conical subvariety of $\mathfrak{s p}(V)^{(1)}=S^{3} V$ :

$$
C_{J}(\mathfrak{s p}(V)):=\left\{S \in C(\mathfrak{s p}(V)) \mid S_{X} J+J S_{X}=0 \quad \text { for all } \quad X \in V\right\}
$$

Lemma 2 Let $S \in C_{J}(\mathfrak{s p}(V))$. Then $\Sigma_{S}$ is $\varnothing$-isotropic and J-invariant (and hence $g$ isotropic).

Proof. This follows immediately from Theorem 9, the equation $J S_{X}=-S_{X} J$ and the definition of the support $\Sigma_{S}$, see equation (2.4).

Now it is easy to prove Theorem 13 .

Proof of Theorem 13. The fundamental tensor field $S=\nabla-D$ associated to a flat special Kähler manifold has values in $C_{J}(\mathfrak{s p}(V))$. We claim that $C_{J}(\mathfrak{s p}(V))=0$ if the metric $g$ is definite. This implies the theorem. By the previous lemma, the support $\Sigma_{S}$ of any $S \in C_{J}(\mathfrak{s p}(V))$ is $g$-isotropic and therefore $\Sigma_{S}=0$ if the metric is definite.

Next we want to describe $C_{J}(\mathfrak{s p}(V))$ for arbitrary signature of the metric. For this it it convenient to complexify $V$. We obtain

$$
V^{\mathbb{C}}:=V \otimes \mathbb{C}=V^{1,0} \oplus V^{0,1}
$$

where $V^{1,0}$ and $V^{0,1}$ are the $\pm i$-eigenspaces of (the complex linear extension of) $J$. The symplectic form $\varnothing$ extends to a complex symplectic form (again denoted by $\varnothing$ ) on $V^{\mathbb{C}}$, for which the decomposition (3.5) is Lagrangian. We have a corresponding type decomposition of the third symmetric power:

$$
S^{3} V^{\mathbb{C}}=\oplus_{p+q=3} S^{p, q} V, \quad S^{p, q} V:=S^{p} V^{1,0} \cdot S^{q} V^{0,1}
$$

Lemma 3 Let $S \in S^{3} V$. Then $S \in C_{J}(\mathfrak{s p}(V))$ if and only if the following two conditions are satisfied 
i) the support $\Sigma_{S}$ of $S$ is $\omega$-isotropic and $J$-invariant.

ii) $S=S_{c}+\bar{S}_{c}$, where $S_{c} \in S^{3,0} V$ is a cubic form of type $(3,0)$ and $\bar{S}_{c}$ is the conjugated cubic form of type $(0,3)$.

Moreover, if $S \in C_{J}(\mathfrak{s p}(V))$ then the support $\Sigma_{S_{c}}$ of $S_{c} \in S^{3,0} V$ satisfies $\Sigma_{S_{c}}=\Sigma_{S}^{1,0}$.

Proof. If $S \in C_{J}(\mathfrak{s p}(V))$ condition i) is satisfied by Lemma 2. To show ii) we consider (see (2.3) $S$ as an element of $S^{3}\left(V^{\mathbb{C}}\right)^{*}=\left(S^{3} V^{\mathbb{C}}\right)^{*}$. We put $V_{1,0}=\left(V^{0,1}\right)^{\wedge}$ and $V_{0,1}=\left(V^{1,0}\right)^{\wedge}$. We consider the induced dual decomposition

$$
S^{3}\left(V^{\mathbb{C}}\right)^{*}=\oplus_{p+q=3} S_{p, q} V, \quad S_{p, q} V:=S^{p} V_{1,0} \cdot S^{q} V_{0,1} .
$$

The condition that $S_{X}$ and $J$ anticommute is expressed by the condition $S(J X, Y, Z)=$ $S(X, J Y, Z)=S(X, Y, J Z)$, for all $X, Y, Z \in V$. This is equivalent to

$$
S \in\left(S^{2,1} V \oplus S^{1,2} V\right)^{\wedge}=S_{3,0} V \oplus S_{0,3} V .
$$

Under the identification via $\omega$ this amounts to $S \in S^{3,0} V \oplus S^{0,3} V$. Complex conjugation on $V^{\mathbb{C}}$ extends to an antilinear involution $\rho$ on $S^{3} V^{\mathbb{C}}$ so that $S^{3} V=\left(S^{3} W^{\mathbb{C}}\right)^{\rho}$ is the fixed point set of $\rho$. Since $\rho$ interchanges $S^{3,0} V$ and $S^{0,3} V$, ii) holds. For the converse, we note that condition i) implies (see Lemma 1) that $S \in C(\mathfrak{s p}(V)$ ). We just saw that condition ii) implies that $S_{X}$ and $J$ anticommute. Therefore i) and ii) imply $S \in C_{J}(\mathfrak{s p}(V))$.

We compute now the support $\Sigma_{S_{c}} . S_{c} \in S^{3,0} V=S^{3} V^{1,0}$ implies that $\Sigma_{S_{c}} \subset V^{1,0}$, and correspondingly we get $\Sigma_{\bar{S}_{c}} \subset V^{0,1}$. Since

$$
\left(\Sigma_{S}\right)^{\mathbb{C}}=\left(\Sigma_{S}\right)^{1,0} \oplus\left(\Sigma_{S}\right)^{0,1}=\Sigma_{S_{c}}+\Sigma_{\bar{S}_{c}}
$$

we conclude that $\Sigma_{S_{c}}=\Sigma_{S}^{1,0}$.

Let $W$ be a $J$-invariant isotropic subspace of $V$. Let us put

$$
S^{3,0} W^{\rho}:=\left\{S_{c}+\bar{S}_{c} \mid S_{c} \in S^{3,0} W\right\}=\left(S^{3,0} W+S^{0,3} W\right)^{\rho} \subset S^{3} W .
$$

The previous lemma implies the following theorem.

\section{Theorem 14}

$$
C_{J}(\mathfrak{s p}(V))=\bigcup_{U} S^{3,0} U^{\rho} \subset S^{3} V
$$

where the union is over all isotropic complex subspaces $U \subset V$ of maximal dimension. 
Now we study more closely the cone $C_{J}(\mathfrak{s p}(V))$. Let us define

$$
C_{J}(\mathfrak{s p}(V))_{k}:=C_{J}(\mathfrak{s p}(V)) \cap C(\mathfrak{s p}(V))_{k} .
$$

We denote by $\mathbb{G}_{k}(V)$ the Grassmannian of complex subspaces $W \subset V$ of (complex) dimension $k$ and by $\mathbb{G}_{k}^{0}(V) \subset \mathbb{G}_{k}(V)$ the real submanifold which consists of isotropic subspaces. Let $\mathbb{U}_{k} \rightarrow \mathbb{G}_{k}^{0}(V)$ be the universal (tautological) vector bundle. It is the restriction of the holomorphic universal bundle of the complex Grassmanian $\mathbb{G}_{k}(V)$ to the submanifold $\mathbb{G}_{k}^{0}(V) \subset \mathbb{G}_{k}(V)$.

Theorem 15 The cone $C_{J}(\mathfrak{s p}(V))$ has a stratification:

$$
C_{J}(\mathfrak{s p}(V))=\bigcup_{k=0}^{N} C_{J}(\mathfrak{s p}(V))_{k},
$$

where the number $N \leq n / 2$ is the maximal complex dimension of an isotropic complex subspace of $V$. It is given by $N=\min (p, q)$ if $g$ has complex signature $(p, q), p+q=n=$ $\operatorname{dim}_{\mathbb{C}} V$. The $k$-th stratum

$$
C_{J}(\mathfrak{s p}(V))_{k}=\bigcup_{W \in \mathbb{G}_{k}^{0}(V)} S^{3,0} W_{r e g}^{\rho} \cong \bigcup_{W \in \mathbb{G}_{k}^{0}(V)} S^{3,0} W_{\text {reg }}
$$

is identified with a Zariski open subbundle of the vector bundle $S^{3} \mathbb{U}_{k}$. The action of $\operatorname{Aut}(V, J, \varnothing)=\operatorname{Aut}(V, J, g)=\mathrm{U}(p, q)$ on $C(\mathfrak{s p}(V))$ preserves the stratification and we have the following identification of orbit spaces:

$$
C_{J}(\mathfrak{s p}(V))_{k} / \mathrm{U}(p, q)=S^{3,0} W_{r e g}^{\rho} / \mathrm{GL}_{\mathbb{C}}(W),
$$

where $W \subset V$ is a fixed complex $k$-dimensional isotropic subspace.

Proof. This follows from Theorem 10 and Theorem 14, taking under consideration the canonical identification $S^{3,0} W^{\rho} \stackrel{\sim}{\rightarrow} S^{3,0} W, S_{c}+\bar{S}_{c} \mapsto S_{c}$.

\subsection{Local characterization of flat special special Kähler mani- folds}

Now we derive classification results for indefinite special Kähler manifolds. As before, let $(V, J, g)$ be a standard (pseudo-) Hermitian vector space of complex signature $(p, q)$, $p+q=n$. 
Theorem 16 Let $f$ be a holomorphic function defined on an open subset $M \subset V^{*}$. Assume that the pointwise support of the cubic tensor field $\partial^{3} f: M \rightarrow S^{3,0} V^{*}$ defined by the holomorphic third partial derivatives of $f$ is isotropic and put $S_{f}:=\partial^{3} f+\overline{\partial^{3} f} \in$ $S^{3} V^{*} \cong S^{3} V$. Then $M_{f}:=\left(M, J, g, \nabla:=D+S_{f}\right)$ is a flat special Kähler manifold and any flat special Kähler manifold arises locally in this way.

Proof. Let $f$ be a holomorphic function such that $\partial^{3} f$ has isotropic support. Then, by Lemma 3, the cubic tensor field $S_{f}:=\partial^{3} f+\overline{\partial^{3} f} \in S^{3} V$ has also isotropic support. This implies condition (ii) of Proposition 1 . Condition (iii) follows essentially from the construction of $S_{f}$ by means of partial derivatives, as we show next. We compute for constant (with respect to $D$ ) vector fields $X, Y, Z, W \in V$ :

$$
\begin{aligned}
\left(D_{X} S\right)(Y, Z, W) & =X S(Y, Z, W) \\
& =\frac{1}{8} X Y^{1,0} Z^{1,0} W^{1,0} f+\frac{1}{8} X Y^{0,1} Z^{0,1} W^{0,1} \bar{f} \\
& =\frac{1}{16} X^{1,0} Y^{1,0} Z^{1,0} W^{1,0} f+\frac{1}{16} X^{0,1} Y^{0,1} Z^{0,1} W^{0,1} \bar{f}
\end{aligned}
$$

where

$$
X^{1,0}:=\frac{1}{2}(X-i J), \quad X^{0,1}:=\frac{1}{2}(X+i J) .
$$

This shows that the tensor $D S$ is completely symmetric, which is the content of (iii) in Proposition [. Finally, (iv) is a direct consequence of Lemma 3 and the definition of $C_{J}(\mathfrak{s p}(V))$. So we have checked that $S_{f}$ satisfies the conditions (i-iv) of Proposition t and, hence, it defines a flat special Kähler manifold.

Conversely, by Proposition \&, any flat special Kähler manifold $(M, J, g, \nabla)$ is locally determined by the tensor field $S=\nabla-D: M \rightarrow C_{J}(V)$, for which $D S$ is completely symmetric. This is the integrability condition for the existence of a function $h$ such that $C=D^{3} h$. From Lemma 3 it follows that $S=S_{c}+\bar{S}_{c} \in S^{3,0} V+S^{0,3} V$ and the complete symmetry of $D S$ implies the complete symmetry of $D^{1,0} S_{c}$ and $D^{0,1} \bar{S}_{c}$, where $D=D^{1,0}+D^{0,1}$ is the type decomposition of the (complexified) flat torsionfree connection $D$. This shows the existence of a holomorphic function $f$ such that $S_{c}=\partial^{3} f$, where $\partial:=D^{1,0}$. Thus, $S=S_{f}$. We know by Lemma 2 that the support of $S$ is isotropic and, hence, by Lemma 3 , also the support of $S_{c}$ is isotropic. We remark that the holomorphic function $f$ is related to the real function $h$ in the proof by $h=8(f+\bar{f})$.

Remark 1 It follows from Proposition t that the tensor field $S=\nabla-D=S_{f} \in S^{3} V$ associated to a flat special Kähler manifold defines by means of $\varnothing$ a commutative and associative multiplication $X \circ Y:=S_{X} Y$ on each tangent space. Moreover $S$ is potential 
in the sense that $S$ is defined by the third derivatives of the function $h=8(f+\bar{f})$ with respect to the flat torsionfree symplectic (and metric) connection $D$. This is similar to the type of structure one encounters in the theory of Frobenius manifolds. In that theory (see e.g. [D]) one also has a cubic tensor field $S$ which is a section of $S^{3} T^{*} M$ and which is identified with a commutative and associative multiplication by means of an isomorphism $T^{*} M \cong T M$. However, the isomorphism is given by a flat metric, whereas in our case we use the symplectic structure. In both cases $S$ is potential with respect to a flat torsionfree connection compatible with the identification $T^{*} M \cong T M$.

\subsection{Examples with constant cubic form}

Let $(M, J, g, \nabla)$ be a special Kähler manifold. We say that it has constant cubic form if $D S=0$, where $S=\nabla-D$. The special Kähler manifold $M_{f}$ in the above theorem has constant cubic form if and only if $f$ is a cubic polynomial. In that case we may assume that $f$ is a homogeneous polynomial, since lower order terms are annihilated by third derivatives. Notice that if $f$ is a homogeneous polynomial then the condition on the third derivatives is satisfied if and only if $f$ considered as an element of $S^{3} V$ has isotropic support. We say that a special Kähler manifold $(M, J, g, \nabla)$ has a trivial factor if it is the product of two special Kähler manifolds and one of the factors is trivial.

Lemma 4 The flat special Kähler manifold $(V, J, g, \nabla=D+S)$ defined by a cubic form $S \in C_{J}(\mathfrak{s p}(V))$ has a trivial factor if and only if $\operatorname{dim} \Sigma_{S}<n=\frac{1}{2} \operatorname{dim} V$.

Proof. Suppose that $(V, J, g, \nabla=D+S)$ has a trivial factor. Then there exists an orthogonal and complex splitting $V=V_{0} \oplus V_{1}$, where $V_{0} \subset \operatorname{ker} S$ is tangent to the trivial factor. Therefore $\Sigma_{S}$ is contained in the nondegenerate subspace $V_{1}$. This implies that the isotropic subspace $\Sigma_{S} \subset V_{1}$ has dimension $\operatorname{dim} \Sigma_{S} \leq \frac{1}{2} \operatorname{dim} V_{1}<n$. Conversely, if the isotropic space $W=\Sigma_{S}$ has dimension $\operatorname{dim} W<n$ then we may choose an isotropic complement $W^{\prime}$ of $W$ which satisfies $\operatorname{dim} W^{\prime}=\operatorname{dim} W$, so that $V_{1}=W \oplus W^{\prime}$ is a nondegenerate subspace. Putting $V_{0}=V_{1}^{\perp}$ we get a complex orthogonal decomposition $V=V_{0} \oplus V_{1}$, with $V_{0} \subset \operatorname{ker} S$. This shows that $V_{0}$ with the induced structures is a trivial factor of $(V, J, g, \nabla=D+S)$.

It follows from the Lemma and the remark above that any flat special Kähler manifold $M$ without a trivial factor and with constant cubic form is locally of the form $M_{f}$, where $f \in S^{0,3} U \subset S^{0,3} V \cong S^{3,0} V^{*}$ and $\Sigma_{S}=U \subset V$ is a complex Lagrangian subspace. In 
particular the Hermitian vector space $(V, J, g)$ has real signature $(2 k, 2 k)$, and $\operatorname{dim} M=$ $4 k$.

Theorem 17 Let $(V, J, g)$ be a Hermitian vector space which admits a complex Lagrangian subspace $U$, and let $f \in S^{0,3} U_{\text {reg }} \subset S^{0,3} V \cong S^{3,0} V^{*}$. Then the manifold $M_{f}$ associated to the holomorphic cubic polynomial function $f: M=V \rightarrow \mathbb{C}$ is a flat special Kähler manifold without trivial factor, with constant cubic form and complete connections $D$ and $\nabla$. Conversely every flat special Kähler manifold without trivial factor and with constant cubic form is locally of this form. Moreover, the correspondence $f \mapsto M_{f}$ defines a bijection between the orbits of the group $\mathrm{GL}_{\mathbb{C}}(U)$ on $S^{0,3} U_{\text {reg }}$ and germs of flat special Kähler manifolds with constant cubic form and without trivial factor up to equivalence.

Proof. We already remarked that flat special Kähler manifolds without trivial factor and constant cubic form are locally of the form $M_{f}$, for $f \in S^{0,3} U_{\text {reg }}$. Two germs of manifolds $M_{f}$ and $M_{f^{\prime}}$ are isomorphic, if and only if there exists a germ of holomorphic isometry, i.e. an affine transformation with linear part in $\operatorname{Aut}(V, J, g)=U(m, m)$ which maps the tensor $S_{f}$ to $S_{f^{\prime}}$. In other words $M_{f}$ and $M_{f^{\prime}}$ are isomorphic if and only if $f$ and $f^{\prime}$ are equivalent under the (pseudo-) unitary group $\mathrm{U}(m, m)$. From Theorem 15 (3.7) we have the identification

$\bigcup_{W \in \mathbb{G}_{m}^{0}} S^{3} W_{r e g}^{\rho} / \mathrm{U}(m, m)=C_{J}(\mathfrak{s p}(V))_{m} / \mathrm{U}(m, m) \cong S^{3,0} U_{r e g}^{\rho} / \mathrm{GL}_{\mathbb{C}}(U) \cong S^{0,3} U_{\text {reg }} / \mathrm{GL}_{\mathbb{C}}(U)$, where the union is over all complex Lagrangian subspaces $W \subset V$. (They have complex dimension $N=m=n / 2$.) This shows that the correspondence $S^{0,3} U_{\text {reg }} / \mathrm{GL}_{\mathbb{C}}(U) \ni f \mapsto$ $M_{f}$ onto isomorphism classes of germs of flat special Kähler manifolds with constant cubic form and without trivial factor is one-to-one. Note that $D$ is the canonical complete connection on the vector space $M=V$. We explain now why $\nabla$ is complete. Recall from section 2 that by iii) of Proposition 2 the tensor $S=D-\nabla \in C(\mathfrak{s p}(V))$ defines a simply transitive affine action of $V$ on itself. The orbit map $\Phi$ in $0, V \ni X \mapsto X+S_{X} X \in V$, of this action is therefore a diffeomorphism and it is easy to very that $\nabla=\Phi^{*} D$. Hence, in particular $\nabla$ is complete.

Remark 2 The flat special Kähler manifolds $M_{f}=\left(V, J, g, \nabla=D+S_{f}\right)$ of Theorem 17 admit a simply transitive vector group of automorphisms. In fact, we can consider the constant tensor fields $J, g$ and $S_{f}$ as left-invariant complex structure, metric and connection on the vector group $V$. Every flat special Kähler manifold with a simply transitive group of automorphisms is of this type. 
By the c-map, see [ACD and references therein, we can associate with the special Kähler manifold $M_{f}$ a flat hyper-Kähler manifold of signature $(4 m, 4 m)$. This flat hyper-Kähler manifold then admits a simply transitive group of automorphisms which is a semi-direct product of two vector groups of dimension $4 \mathrm{~m}$.

Remark 3 The examples $M_{f}=\left(V, J, g, \nabla=D+S_{f}\right)$ are complete with respect to both connections $\nabla$ and $D$. It would be interesting to know if there do exist further examples of flat special Kähler manifolds (i.e. examples with a non-constant cubic form) which are complete with respect to both connections.

\section{References}

[A] L. Auslander, Simply Transitive Groups of Affine Motions, Amer. J. Math., 99 (1977), no. 4, 809-826

[ACD] D. V. Alekseevsky, V. Cortés, C. Devchand, Special complex manifolds, to appear in J. Geom. Phys., math.DG/9910091

[BC] O. Baues and V. Cortés, Realisation of special Kähler manifolds as parabolic spheres, Proc. Amer. Math. Soc., 129 (2001), no. 8, 2403-2407

[Ca] E. Calabi, Improper affine hypersurfaces of convex type and a generalization of a theorem of Jörgens, Michigan Math. J. 5 (1958), 105-126

[D] B. Dubrovin, Geometry of $2 D$ topological field theories, in Integrable systems and quantum groups (Montecatini Terme, 1993), Lecture Notes in Math. 1620, 120348, Springer, 1996

[DO] K. Dekimpe, V. Ongenae, On the number of abelian left symmetric algebras, Proc. Amer. Math. Soc. 128 (2000), no. 11, 3191-3200

[F] D. S. Freed, Special Kähler manifolds, Commun. Math. Phys. 203 (1999), no. 1, $31-52$

[FGH] D. Fried, W. Goldman, M.W. Hirsch, Affine manifolds with nilpotent holonomy, Comment. Math. Helv. 56 (1981), no. 4, 487-523.

[K] S. Kobayashi, Transformation groups in differential geometry, Springer-Verlag, 1972 
[L] Z. Lu, A note on special Kähler manifolds, Math. Ann. 313 (1999), no. 4, 711-713

[NS] K. Nomizu, T. Sasaki, Affine differential geometry. Geometry of affine immersions, Cambridge Tracts in Mathematics 111, Cambridge University Press, Cambridge, 1994

[S] D. Segal, The structure of complete left-symmetric algebras, Math. Ann. 293 (1992), no. 3, 569-578

[VLS] L. Vrancken, A. M. Li, U. Simon, Affine spheres with constant affine sectional curvature, Math. Z. 206 (1991), no. 4, 651-658 